\title{
El aumento de la escolarización en Educación Infantil y la disminución de las tasas de abandono temprano: dos indicadores que favorecen las políticas de inclusión
}

\author{
Sánchez Lissen,Encarnación \\ Universidad de Sevilla, Sevilla, España \\ eslissen@us.es
}

\section{Resumen}

No se entiende hoy un sistema educativo de calidad si no incluye políticas encaminadas a mejorar las oportunidades educativas de todos los estudiantes.

En todos los casos, los sistemas deben evolucionar favoreciendo las características personales y, a la vez, integrando las diferencias económicas, sociales, culturales, religiosas de cada país. Esta pluralidad solo tendrá verdadero sentido si se presentan como oportunidades y, en definitiva, como un camino necesario para lograr la inclusión, para mejorar y evolucionar.

Para que cada sociedad alcance una mayor igualdad, tanto la educación como la formación juegan un papel determinante. En la Unión Europea, la Comisión en materia de educación y formación ha propuesto, de cara al 2020, la consecución de seis objetivos. Entre ellos se anuncia la importancia que tiene mejorar la escolarización de las etapas de educación infantil así como las tasas de abandono prematuro. Concretamente, en el próximo bienio, las cifras de niños y niñas en edad preescolar de más de cuatro años deben alcanzar el $95 \%$ y, por otro lado, se debe lograr reducir por debajo del 10\%, el abandono temprano de escolares en la educación y la formación.

Este estudio analiza estos dos indicadores, viendo su evolución en diversos países y reconociendo la relevancia que pueden tener ambos, ya directa o indirectamente, en los procesos de inclusión y en su optimización. Aumentando el primero y disminuyendo el segundo se favorecen las medidas relacionadas con la proporcionalidad y la equidad educativa. La elección de estos dos ámbitos tiene su explicación en el propio concepto de inclusión y en las dimensiones asociadas al mismo.

¿Sería acertado indicar que aquellos países que tienen mejores cifras en materia de educación infantil y de abandono temprano tienen también mejores datos acerca de la calidad educativa y, por consiguiente, en materia de inclusión? Buscamos algunos ejemplos que nos ayuden a entender y explicar estos argumentos aunque, realmente, no parece oportuno considerarlos como hechos absolutos pero sí relacionados.

\section{Abstract}

A quality education system needs to include policies aimed at improving the educational opportunities of all students. The development of education systems should promote personal characteristics in all cases while, at the same time, integrating the economic, social, cultural, and religious differences within each country. This diversity will only have real meaning if it is presented as an opportunity and ultimately, as a necessary inclusionary path for improvement and growth.

Education and training play a decisive role in achieving greater social equality. In the European Union, the Education and Training Commission has proposed six benchmarks to be met by 2020 . These include improving schooling provision for the early stages of primary education, as well as improving early drop-out rates. Specifically, in the next two years, at least $95 \%$ of children (from 4 to compulsory school age) should participate in early childhood education school; and the rate of early leavers from education and training aged 18-24 should be below $10 \%$. This study analyses these two indicators by looking at how they have fared in different countries and recognising their direct or indirect impact in the optimisation of inclusion processes. Increasing the first indicator while decreasing the second encourages measures related to proportionality and educational equality. The choice of these two areas can be explained by the concept of inclusion and its associated dimensions.

Would it be accurate to say that those countries that have better figures in terms of early childhood education and early school drop-out rates also have better data in terms of educational quality and, consequently, of inclusion? This paper provides some examples to gain a better understanding of these arguments, since it does not seem appropriate to consider them in isolation but rather as interrelated aspects.

Palabras clave: políticas de inclusión, educación infantil, abandono temprano, equidad, oportunidades educativas.

Keywords: inclusion policies, early childhood education, drop-out rates, equality, educational opportunities. 


\section{INTRODUCCIÓN}

No se entiende hoy un sistema educativo de calidad si no incluye políticas encaminadas a mejorar las oportunidades educativas de todos los estudiantes.

En todos los casos, los sistemas deben evolucionar favoreciendo las características personales y, a la vez, integrando las diferencias económicas, sociales, culturales e incluso religiosas de cada país. Esta pluralidad solo tendrá verdadero sentido si se presentan como oportunidades y, en definitiva, como un camino necesario para lograr la inclusión, para mejorar y evolucionar. Concretamente, la propuesta de la Comisión Europea para actualizar la Recomendación del Parlamento y del Consejo sobre Competencias Clave para el Aprendizaje Permanente (2012) describe, en un documento titulado: Un nuevo concepto de educación: invertir en las competencias para lograr mejores resultados socioeconómicos, cuáles son los compromisos más sugerentes para obtener esos nuevos logros.

Para desarrollar esas competencias se advierte de la necesidad de atender y cumplir unos requisitos mínimos que favorezcan el crecimiento educativo en cada país. Con este reto, la Comisión en materia de educación y formación de la Unión Europea señala que, entre las medidas políticas de peso que se deben implementar para alcanzar la excelencia educativa, se encuentran los siguientes objetivos. Estos son (Comisión Europea, 2017:2):

1. Reducir por debajo del $10 \%$ el abandono prematuro de la educación y la formación.

2. Llegar a una cuota del $40 \%$ de educación superior entre la población de 30 a 34 años.

3. Alcanzar el $95 \%$ de participación en educación y cuidados de la primera infancia.

4. Reducir por debajo del $15 \%$ el bajo rendimiento en lectura, matemáticas y ciencias.

5. Alcanzar un índice de empleo del $82 \%$ entre los titulados recientes.

6. Llegar a una cuota del $15 \%$ de participación de los adultos en el aprendizaje.

Sabiendo el valor que representa de cara a la educación de un país cada uno de estos indicadores y estas cifras, apreciamos que, en este proceso de cambio, de mejora y de apertura, las políticas de inclusión, abrigadas por la mayoría de gobiernos, procederán a gestionar de una manera especial el valor de la equidad en el ámbito formal de la educación. Éste será el pilar que fundamenta los dos indicadores centrales de este artículo: potenciar la educación infantil y reducir el abandono escolar. En él se analizan dichos indicadores, viendo su evolución en diversos países y reconociendo la relevancia que pueden tener ambos, ya directa o indirectamente, en los procesos de inclusión y en su optimización. Aumentando el primero y disminuyendo el segundo se favorecen las medidas relacionadas con la proporcionalidad y la equidad educativa. La elección de estos dos ámbitos tiene su explicación en el propio concepto de inclusión y en las dimensiones asociadas al mismo.

A priori podríamos pensar que aquellos países con mejores cifras en materia de educación infantil y de abandono temprano, también tendrán mejores datos en lo que respecta a la calidad educativa y, por consiguiente, serán mejores los valores relacionados con la inclusión. Sin embargo, a pesar de su importancia y de su posible relación, no parece oportuno considerarlos como hechos absolutos aunque sí relacionados.

Por tanto, tres aspectos conformarán nuestro artículo. En primer lugar, el concepto y valoración de la equidad, a continuación, las cifras que se manejan en torno a los dos indicadores y, en tercer lugar, analizaremos las tendencias, propuestas y beneficios que puede alcanzar la educación de un país, mejorando significativamente ambos factores.

\section{EN EL HORIZONTE, LA EQUIDAD}

En términos educativos hablar de equidad es hablar de calidad, de excelencia y de inclusión. Es un pilar fundamental para la construcción de una persona y de todo un sistema. En realidad, es operativo vincular los conceptos de equidad y calidad, dado que el primero contribuye a crear situaciones de éxito, de superación y previene, en muchos casos, las situaciones de fracaso y abandono escolar (OECD, 2012). 
Asimismo, el concepto de educación inclusiva resalta unido al de equidad, al de una educación para todos. Es un concepto que comenzó a fraguarse en la segunda mitad del siglo XX y que ha dado pasos sustanciales en el siglo XXI, complementado con experiencias, investigaciones y proyectos que nos han llevado a valorarlo ampliamente. Entre otros, pensando en la inclusión, fueron hitos significativos los objetivos comprometidos en la Conferencia Mundial sobre Educación Para Todos (EPT, 1990), los Objetivos de Desarrollo del Milenio (ODM, 2000), las claves de la Convención sobre los Derechos del Niño (CDN, 1989) o el resto de documentos de la UNESCO, de ONGs o de Organismos Nacionales e Internacionales.

Como podemos apreciar, aunque el concepto es amplio, para este trabajo nos aproximamos a la idea de igualdad de oportunidades de aprendizaje, desarrollo de potencialidades, al sentido de justicia social o al de bienestar social.

La importancia que está adquiriendo este término lo podemos comprobar en la aportación que realizan Sánchez-Santamaría, J., y Manzanares, A. (2012) sobre las revistas científicas de impacto que incorporan artículos sobre esta cuestión. Esta profusión de publicaciones vierte sobre el tema un halo de interés notable. De hecho, en estos momentos, la equidad educativa se ha convertido en una cuestión de primer orden en las agendas políticas sobre educación de muchos países (OECD, 2012; Sánchez y Manzanares, 2014). Nos aproximamos al concepto a través de Sicilia y Simancas (2018:9), al argumentar que: un sistema educativo se considera equitativo cuando es capaz de atenuar las desigualdades socioeconómicas existentes en la población, de tal forma que ofrezca a los estudiantes igualdad de oportunidades en el acceso a una educación de calidad y les garantice que su rendimiento académico vendrá determinado por su esfuerzo y capacidad, independientemente de su contexto social, económico y familiar.

Desde el ya conocido Informe Coleman (Coleman et al., 1966), un amplio número de investigaciones han demostrado que el origen social o el nivel socioeconómico inciden directamente en el rendimiento de los alumnos. A pesar de las limitaciones que alcanza la propia escuela, entendemos que no existe la menor duda para concluir que la educación es una herramienta válida y eficaz que ayuda a reducir esas diferencias que no permiten un desarrollo, al mismo nivel, de todos los estudiantes.

Concretamente, el Informe de la OCDE sobre Equity and Quality in Education (2012) puso de manifiesto que casi uno de cada cinco estudiantes, tiene serias dificultades para alcanzar las competencias mínimas que son necesarias para funcionar en la sociedad de hoy. Esta circunstancia se explica, entre otras cosas, por los problemas de inclusión que tienen. Para comprender este dato podemos acudir a las cifras sobre el abandono escolar y comprobar que en torno al $20 \%$ de los adultos jóvenes, dejan los estudios sin completar la educación media superior (OECD, 2012). Sigue primando en todo ello que los estudiantes de entornos más desfavorecidos y por tanto, de un nivel social y económico inferior, tendrán el doble de probabilidad de tener un rendimiento bajo $(O E C D, 2012)$

Caminar hacia la equidad es una garantía de un buen modelo educativo y de políticas integradoras. En esta línea, la propia OCDE concreta en una de sus publicaciones diez pasos hacia la equidad en educación.

Al echar una mirada a los Informes PISA de la última década, adquirimos algunas claves para comprobar qué países han mejorado la equidad educativa. Para ello se utiliza el Índice Socioeconómico y Cultural (ISEC), cuya denominación y siglas en inglés corresponden a:

Economic, Social and Cultural Status (ESCS). En PISA, y también en PIRLS-TIMSS, este índice se obtiene a partir de los cuestionarios que rellenan los estudiantes, profesorado y familias, en relación al contexto social, económico y familiar. Entre los indicadores se obtiene información sobre el nivel de estudios y profesión de los padres, sobre los recursos tecnológicos, sobre los espacios de trabajo o sobre el conjunto de recursos culturales y libros con los que cuenta el alumnado en su vida diaria.

El valor de cada país se obtiene, básicamente, a partir de la media de los índices de los estudiantes y de manera ponderada, en relación al número de individuos de cada población. Se expresa con una media aritmética de 0 y una desviación típica de 1. Concretamente, los últimos Informes muestran que, en Europa, destacan países como Finlandia, Noruega, Suecia, Alemania, Irlanda del Norte que han alcanzado un valor entre 0, 50 y 0. En España, el índice asignado es prácticamente igual a la media. 
En cualquier caso, tal como reconocen los expertos en este asunto, el entorno influye pero no determina.

Podemos apreciar en el siguiente gráfico, cómo han evolucionado (2006-2015) diversos países, en las cifras de rendimiento y equidad. En este caso se valora el aumento experimentado por parte de países como: Bulgaria, Brasil, Dinamarca, Estados Unidos, Chile, Italia, Letonia, Macao (China), Méjico, Polonia, Rumanía, Tailandia, Turquía y Uruguay. España se sigue situando en un cuadrante en el que se aprecia que aumenta el rendimiento pero decrecen los valores de equidad.

Gráfico 1. Rendimiento y equidad en diversos países.

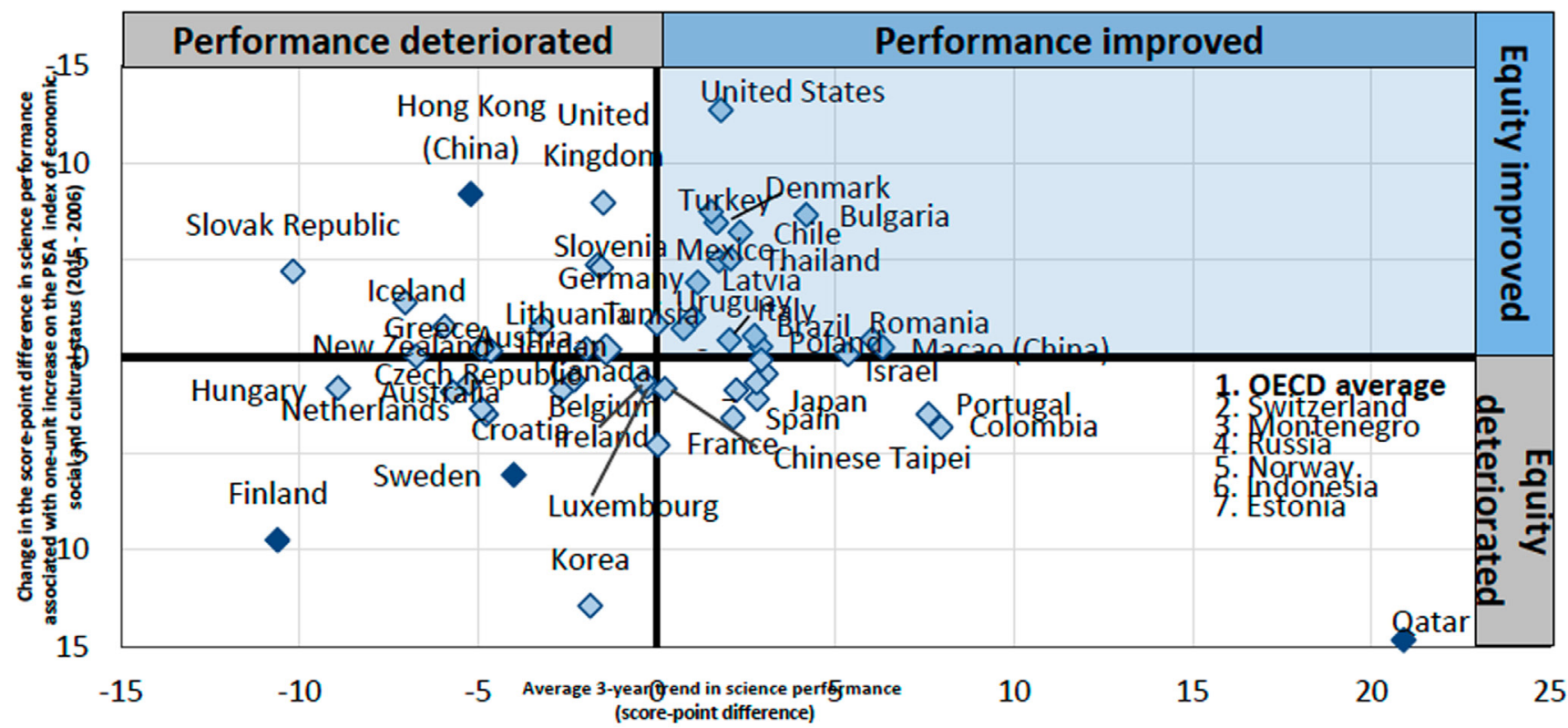

Fuente: OCDE, Base de datos de PISA 2015 (Tabla I.6.17).

A la vista de estos resultados, el propio Informe PISA (2015) señala que una mayor equidad no tiene por qué producirse en detrimento del rendimiento. En este último estudio en el que se analizaba expresamente la competencia de ciencias, se ha puesto de manifiesto que, entre 2006 y 2015 , el grado de desigualdad socioeconómica se redujo en nueve países que, a su vez, lograron mantener su rendimiento medio en ciencias: Brasil, Bulgaria, Chile, Dinamarca, Alemania, Montenegro, Eslovenia, Tailandia y los Estados Unidos. En estos países, la predicción del rendimiento a partir del nivel socioeconómico de los estudiantes se hizo menos fiable, mientras que el rendimiento medio se mantuvo estable (PISA in FOCUS, 2017, 68, p.4).

Con estas consideraciones previas sobre el valor de la equidad y el sentido de la inclusión, analizamos cómo pueden influir algunos indicadores en la evolución y desarrollo de los mismos.

\section{DISMINUYENDO LAS TASAS DE ABANDONO TEMPRANO}

Viene siendo habitual que aquellos países que tienen malas cifras de fracaso y abandono escolar, presentan también cifras mediocres en temas relacionados con el rendimiento de los estudiantes. Sin embargo, también en este asunto la equidad juega un papel importante. Concretamente, en esta proposición, tiene mucho que ver tanto, el nivel de desigualdad que tenga ese país en materia educativa, como los niveles mínimos de competencias que se adquieran. De hecho, estudios como el de Sicilia y Simancas (2018:30) argumentan que «la pobreza educativa está fuertemente asociada al fracaso escolar y al abandono temprano, dos problemas recurrentes en el sistema educativo español». Si mejoramos los valores en materia de escasez educativa podremos contribuir a mejorar también los valores asociados a la permanencia en el sistema. Culminar un proceso, una vez alcanzadas las competencias mínimas, le traslada seguridad, rigor, logros y formación útil a los candidatos. 
En la actualidad, las cifras marcan una tendencia más favorecedora que, a medio o corto plazo deben satisfacer los modelos educativos de cada país. En España, el abandono escolar temprano se ha reducido ligeramente en relación con el año pasado. Concretamente, el porcentaje es del 18,3\% lo que supone siete décimas menos que el año anterior. A pesar de estos datos, las diferencias entre las Comunidades Autónomas son notables ya que, si bien cae en regiones como Aragón, Murcia o La Rioja, sin embargo, suben unos puntos más en Cantabria, Valencia y Andalucía.

A nivel internacional se ha clasificado a Sudán del Sur como el país que tiene la mayor tasa de abandono escolar del mundo: 76\% (UNICEF, 2017).

Para medir el abandono, debemos conocer el porcentaje de población que no haya culminado la Educación Secundaria de segunda etapa y no continúe ningún tipo de estudios o de formación complementaria. Este dato se puede analizar unido a las tasas de graduación que, a su vez, proporciona información sobre el número de estudiantes que han abandonado el sistema educativo sin finalizar un proceso de cualificación completo. Si analizamos cuáles son los factores que más influyen en el abandono escolar podemos sugerir los siguientes: la situación familiar, los aspectos socioeconómicos de su entorno, o bien otros más personales relacionados con las dificultades de aprendizaje, como son: la motivación personal o la cultura del estudio.

En la Unión Europea (UE-28) podemos apreciar que las cifras más altas la siguen manteniendo España, Malta, Rumanía y Turquía. De PISA, los países con mejor posición, tienen también en este punto cifras por debajo del 12\%. Por ejemplo: Estonia: $(10,8)$, Finlandia $(8,2)$, Eslovenia $(4,3)$, Holanda $(7,1)$, Alemania $(8,8)$.

Nos parece llamativo el caso de Croacia $(3,1)$, cuya cifra es la menor. Sin embargo, no se trata de un país bien situado en las escalas de rendimiento ya que en el Informe PISA (2015), se situó en el puesto 38, por debajo de la media de la OCDE y también de España. Este dato nos lleva a entender que la correlación ni es tan estrecha ni es tan directa dado que otros factores marcan e influyen en ambos indicadores.

Gráfico 2. Tasas de abandono escolar en 2017.

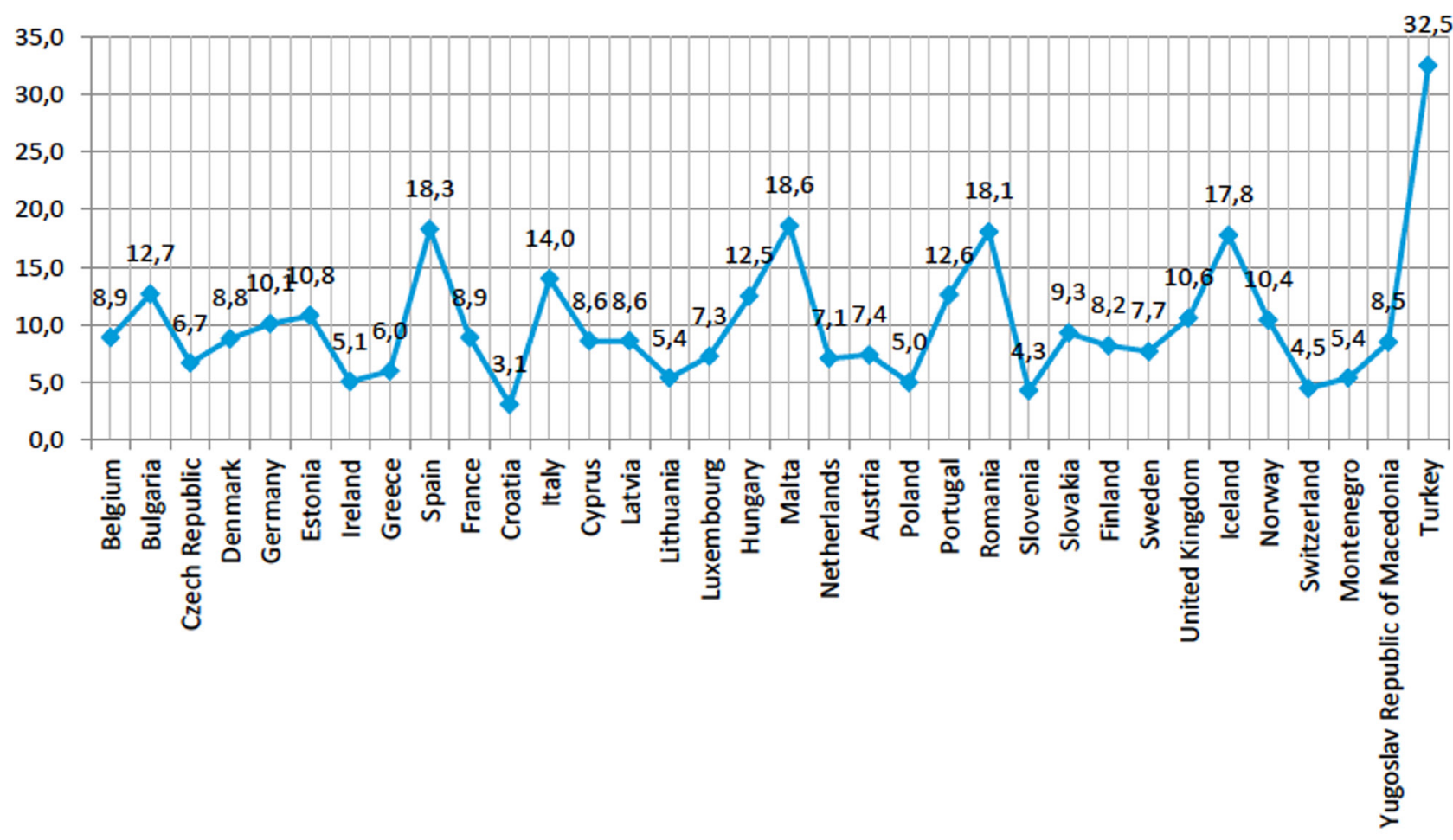

Fuente: Eurostat. Early leavers from education and training by sex and labour status (2017). 


\section{MEJORANDO LAS TASAS DE EDUCACIÓN INFANTIL.}

Cualquier construcción, para que sea segura, efectiva y duradera, es fundamental que sus cimientos sean sólidos. Ciertamente, en el sistema escolar de cualquier país, las primeras etapas representan un enorme baluarte y un apoyo para dar estabilidad a dicha formación. Esta simple proposición tiene, en estos momentos, un valor incontrovertible. En las reformas emprendidas en los países de la OECD (2015) se consigna que el 16\% de dichas medidas se centran en procurar la calidad y la equidad educativa. Pues bien, en este proceso, se procura aumentar la matriculación y mejorar la calidad de las etapas correspondientes a la educación infantil. Este reto avanza en países como Australia y Polonia. En ambos, la atención a la infancia ha mejorado tanto en datos de participación como de calidad.

Realmente, entre los países miembros de la OCDE, los esfuerzos por mejorar la cantidad y calidad de la educación infantil, es un asunto bien arraigado en muchos de ellos. Por ejemplo, el caso de Alemania. Un país que, además, ha recibido un amplio número de refugiados en los últimos años y, entre sus retos ha estado, precisamente, lograr la integración de aquéllos, procurando incrementar las cifras que atañen a las edades infantiles.

Desde este punto de vista, al analizar Estonia, nos llama la atención la falta de correspondencia que existe entre estos datos y su situación en los informes internacionales. Concretamente, el Informe PISA de 2015, sitúa a este país en el tercer lugar del ranking, sin embargo, la participación en la educación infantil y atención a la infancia, se sitúa por debajo de la media de la Unión Europea (Comisión Europea, 2017). A pesar de no estar en consonancia debemos tener en cuenta que, en Estonia, la enseñanza obligatoria se inicia a los siete años y esa circunstancia puede afectar a la matriculación de estudiantes. En cualquier caso, no podemos anticipar que este dato sea negativo de cara a las políticas de educación emprendidas, más bien son potenciadoras de su modelo de educación.

Las propuestas formuladas en diversos países pasan, en muchos casos, por poner en marcha políticas educativas relacionadas con la infancia. Por ejemplo, Bulgaria da cobertura, en los últimos años, a una nueva Ley de Educación Escolar y Preescolar, que propone entre sus retos mejorar las cifras actuales en los que a infancia y escolarización se refiere.

En Hungría, a la vista de los resultados que se han ido obteniendo en los últimos Informes PISA, una de las medidas tomadas han estado en relación con la educación infantil y más concretamente con poner en marcha soluciones que ayuden a disminuir las diferencias entre los resultados de los estudiantes en entornos desfavorecidos y en otros de mayor nivel socioeconómico.

Las cifras que presentan los Países Bajos nos muestra uno de los sistemas educativos más eficaces de la Unión Europea; datos que se hacen patentes en lo que respecta a la participación y calidad de la educación infantil.

Los países iberoamericanos también se han sumado en muchos casos a optimizar estos datos y, aunque aún le queda mucho por mejorar, la Educación y la Atención en la Primera Infancia (EAPI) son un primer paso para el aprendizaje permanente (OECD, 2017, OCDE, 2018). Los alumnos que lo recibieron tienden a mostrar un rendimiento académico mejor en etapas posteriores de su educación.

Es interesante analizar las cifras para valorar este indicador. Realmente, los datos son bastante elevados aunque hay que precisar tanto los países como las etapas a las que se refieren en cada caso. Nos centramos para este estudio en las edades de 2 a 5 años. Se trata de un periodo no obligatorio, en la mayoría de los casos, aunque ello no impide que las tasas de matriculación sean elevadas. La enseñanza comienza a ser obligatoria en la etapa de primaria. Esta se inicia mayoritariamente a los 6 años aunque hay países que comienzan a los 7 años (Bulgaria, Estonia, Croacia, Letonia, Lituania, Polonia, Finlandia y Suecia), algunos menos a los 4 (Irlanda del Norte) y otros a los 5 años (Chipre, Malta, Inglaterra, Gales, Escocia y Turquía). Por tanto, aunque la Educación Infantil podría abarcar algo más de tres años de escolarización, exponemos los datos referidos a las edades de 3 y 4 años.

Concretamente, en la Unión Europea, las cifras en general son altas aunque también hay países que tienen que incrementarlas. 


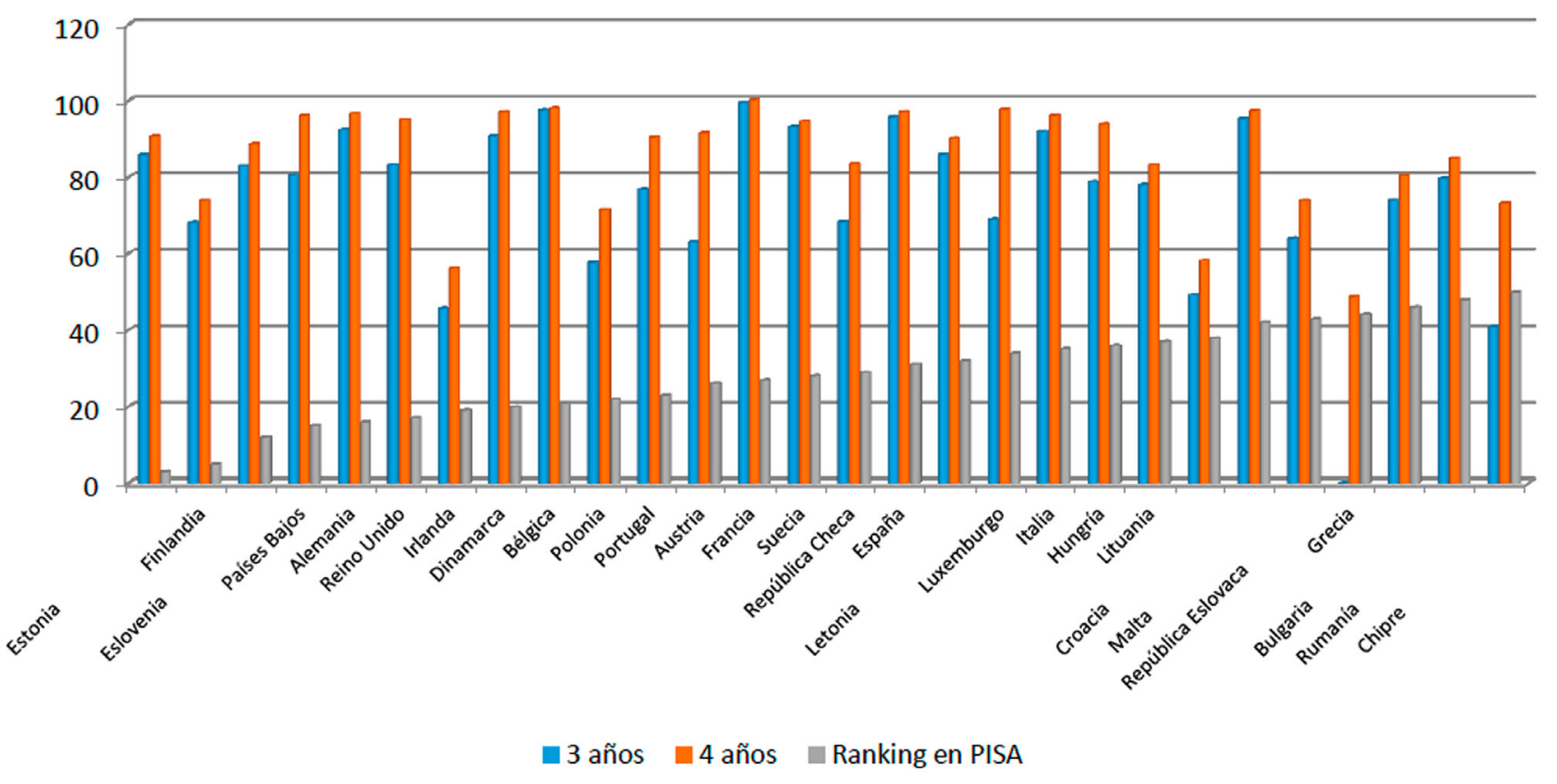

Fuente: Elaboración propia, a partir de Eurostat, 2015 y PISA 2015.

Para corregir estas cifras, un amplio número de países han convenido en considerar a la EAPI como una etapa obligatoria. Este reto se ha iniciado en Europa, en países como Polonia, Austria o Noruega, pero también en otros como Australia, Chile o Argentina. Además de este objetivo, se plantean otras alternativas que ayuden a mejorar los datos, por ejemplo, invertir en el número de plazas disponibles, o mejoras en la dotación de ayudas.

En el gráfico anterior también queda reflejado el lugar que ocupa cada país en el ranking de PISA. Hemos ordenado de izquierda a derecha, los países tal como aparecen en el último Informe de 2015. Como podemos ver, la correlación no es total si analizamos su posición y los valores de escolarización en edad infantil, aunque sí convergen mayoritariamente.

\section{DISCUSIÓN Y CONCLUSIONES}

Para que cada sociedad alcance una mayor igualdad, tanto la educación como la formación juegan un papel determinante. En la Unión Europea, la Comisión en materia de educación y formación ha propuesto, de cara al 2020, la consecución de seis objetivos. Entre ellos se anuncia la importancia que tiene incrementar la escolarización de las etapas de educación infantil así como las tasas de abandono prematuro. Concretamente, en el próximo bienio, las cifras de niños y niñas en edad preescolar de más de cuatro años deben alcanzar el $95 \%$ y, por otro lado, se debe lograr reducir por debajo del $10 \%$, el abandono temprano de escolares en la educación y la formación. Aunque muchos países tienen aún pendiente disminuir y adecuarse a esas cifras, otros ya han alcanzado este cometido. Así lo hemos ido observando a lo largo de este trabajo. En esta línea se articula el Marco Estratégico ET 2020 de la Unión Europea que concreta entre sus objetivos (OE 3) el «promover la equidad, la cohesión social y la ciudadanía activa» (Ministerio de Educación, 2011).

Tomar el pulso de la inclusión versus exclusión y a las políticas favorecedoras de la equidad, no es una tarea sencilla aunque parece cada vez más prioritaria si pretendemos mejorar la calidad educativa. Y, a partir de este objetivo, corregir otros resultados.

Afianzar la formación en las edades iniciales ayuda a regenerar todo el proceso formativo y de desarrollo de los aprendizajes ulteriores. 
Aunque difieran los retos y los recursos empleados, el crecimiento de Europa contribuirá también al crecimiento integral de cada país. En cada caso, y en aras de la calidad educativa, comprobamos que se gestiona de distinta manera y con distinta intensidad, todos aquellos factores que pueden llegar a influir en los resultados de las evaluaciones de la educación.

Combatir la pobreza será un requisito para lograr una sociedad más inclusiva y, de igual manera, combatir la pobreza educativa será un requisito primordial para alcanzar un modelo educativo más inclusivo, más equitativo.

En cualquier caso, todos estos nuevos retos deben estar acompañados de políticas de formación del profesorado que sepan atender ambas estrategias. En buena medida, aunque no sea suficiente, mejorar la cantidad y la calidad del profesorado debe ser un camino necesario para optimizar las cifras que nos ocupan.

\section{REFERENCIAS BIBLIOGRÁFICAS}

Coleman, J. S.; et. al. (1966). Equality of educational opportunity. Washington, U.S.: Government Printing Office.

Comisión Europea (2017). Monitor de la Educación y la Formación de 2017. Volumen II, Dirección General de Educación y Cultura, Comisión Europea. https://ec.europa.eu/education/sites/education/files/monitor2017-es_es.pdf.

Ministerio de Educación (2011). Objetivos Educativos Europeos y Españoles. Estrategia Educación y Formación 2020. Madrid: Secretaría de Estado de Educación y Formación Profesional.

OCDE (2017). PISA in FOCUS\#68. Madrid: OCDE.

OCDE (2018). Competencias en Iberoamérica: Análisis de PISA 2015. OCDE: Fundación Santillana.

OECD (2012). Equity and quality in education. Supporting disadvantaged students and schools.

OECD Publishing, Recuperado de: http://dx.doi.org/10.1787/9789264130852-en.

OECD (2015). Education Policy Outlook 2015: Making Reforms Happen. Paris: OECD Publishing http://dx.doi.org/10.1787/9789264225442-en.

OECD (2017). PISA 2015 Results (Volume III): Students' Well-Being. Paris: OECD Publishing http://dx.doi.org/10.1787/9789264273856-en.

Sánchez-Santamaría, J., y Manzanares, A. (2012). La equidad educativa: dilemas, controversias e implicaciones para garantizar el éxito educativo para todos. En A. Manzanares (coord.), Temas educativos en el punto de mira (pp. 49-75). Madrid: WolterKlawers.

Sánchez-Santamaría, J. y Manzanares, A. (2014). Tendencias internacionales sobre equidad educativa desde la perspectiva del cambio educativo. Revista Electrónica de Investigación Educativa, 16 (1), 12-28. Recuperado de http://redie. uabc.mx/vol16no1/contenido-sanchez-manzanares.htm.

Sicilia, G. y Simancas, R. (2018). Equidad educativa en España: comparación regional a partir de PISA 2015. Madrid: Fundación Ramón Areces y Fundación Europea Sociedad y Educación.

UNICEF (2017). Estado Mundial de la Infancia 2016. Una oportunidad para cada niño. Nueva York: UNICEF. 